\title{
EEG ANALYSIS AND SPECT IMAGING IN ALZHEIMER'S DISEASE, VASCULAR DEMENTIA AND MILD COGNITIVE IMPAIRMENT
}

\author{
Borislav Radić ${ }^{1}$, Ratimir Petrović ${ }^{2}$, Anja Golubić ${ }^{2}$, Ervina Bilić ${ }^{1}$ \& Fran Borovečki ${ }^{1}$ \\ ${ }^{I}$ Department of Neurology, University Hospital Center Zagreb, Zagreb, Croatia \\ ${ }^{2}$ Department of Nuclear Medicine, University Hospital Center Zagreb, Zagreb, Croatia
}

received: 6.11.2017;

revised: 5.9.2018;

accepted: 11.10 .2018

\begin{abstract}
SUMMARY
Background: Alzheimer's disease (AD) and vascular dementia (VaD) represent a leading public-health problem given the rising age of the population. Early diagnosis of dementia, especially at the stage of mild cognitive impairment (MCI) has become an important goal of the modern patient work-up. Brain perfusion single-photon emission computed tomography (SPECT) has become a mainstay of diagnostic algorithms in patients with dementia showing specific patterns of hypoperfusion in temporal and parietal lobes. Clinical electroencephalography (EEG) is a relatively simple and inexpensive diagnostic tool showing potential in assessing cortical thinning and lower perfusion in temporoparoetal regions.

Subjects and methods: Our study was a cross sectional and included retrospective analysis of the group of patients diagnosed with $A D, V a D$ and MCI. The study group consisted of 50 patients - 29 females and 21 males. All patients underwent EEG and SPECT analysis as part of regular work-up.

Result: Patients with AD exhibit EEG changes mostly in the form of theta waves, focal abnormalities and spike-and-wave complexes in frontotemporal regions with the reduction in the amplitude of alpha waves. SPECT in most patients exhibited hypoperfusion in temporoparietal regions with occasional unilateral abnormalities in frontotemporal region. EEG changes in patients with VaD are predominantly in the form of theta waves while SPECT findings show mostly "patchy" abnormalities. EEG readings are normal or exhibit minimal changes in the group of patients with MCI, while SPECT imaging exhibits mostly normal CBF.

Conclusion: EEG and SPECT are diagnostic methods which show specific changes, especially in AD. EEG can be used to monitor the therapeutic effect and progression of $A D$ as well as the possible transition from MCI to early stage AD. SPECT on the other hand, being a more expensive and less available method, can be utilized as an add-on method to increase the specificity and sensitivity of the diagnostic algorithm.
\end{abstract}

Key words: dementia - Alzheimer's disease - EEG analysis - SPECT imaging

\section{INTRODUCTION}

Dementia encompasses a wide range of symptoms associated with a decline in memory or other cognitive skills severe enough to reduce a person's ability to perform everyday activities. Stigmatization of dementia patients hinders early diagnosis and treatment which could improve the course of the illness and create a "better everyday life" with a more inclusive environment for the affected people (Psota 2015, Weichbold 2015). Alzheimer's disease (AD) accounts for 60 to 80 percent of the dementia cases in persons over 65 years of age. AD nowadays represents a leading public-health problem given the rising age of the population, being the most common of all dementias with a steadily increasing incidence and prevalence (Wancata et al. 2015). AD is manifested by progressive deterioration of cognitive functions and characteristic appearance of extracellular senile plaques, deposits of $\beta$-amyloid, and intracellular neurofibrillary tangles in the cortex and subcortical gray matter (Blenow 2006, Burns 2009). Vascular dementia $(\mathrm{VaD})$ is the second most common cause of dementia in older people. Given its variable presentation, it's may be difficult to diagnose, leading to variable projections of incidence and prevalence. Current estimates attribute $15 \%$ to $20 \%$ of dementia cases in older adults to vas- cular dementia (Roman 2002). Avoiding and controlling risk factors such as diabetes, high blood pressure, smoking, and high cholesterol can help curb the risk of $\mathrm{VaD}$. Mild cognitive impairment (MCI) is an intermediate stage between the expected cognitive decline of normal aging and the more-serious decline of dementia. It can involve problems with memory, language, thinking and judgment that are greater than normal agerelated changes. MCI may increase the risk of later progression to dementia, either AD or other dementias. However, some people with MCI never progress to the stage of dementia and few eventually get better.

Clinical electroencephalography (EEG) is a relatively simple and inexpensive diagnostic tool with a high sensitivity for diffuse organic brain damage of various causes and with mounting evidence about the specificity for the type of dementia (Garn et al. 2017). The highest sensitivity is shown in correlation with DAT in Parkinson dementia, showing high degree of EEG abnormality in connection to the disease severity. Dementia with predominantly frontal pathology show less EEG abnormality and EEG is often normal. It is well known that decreases of alpha and beta power and increases of the delta and theta frequencies are related to brain pathology and general cognitive decline (Dierks et al. 2003, Kwak 2006). EEG analysis may be helpful in the 
dementia context because EEG can be used in prediction of the future cognitive decline or conversion to dementia in MCI subjects with high accuracy and quantification of EEG may provide a method for monitoring therapeutic effects. Recent studies suggest that EEG analysis can be used to distinguish AD from other dementias (Cichocki et al. 2005, DeKosky et al. 2003).

Brain perfusion single-photon emission computed tomography (SPECT) imaging is a functional nuclear imaging technique performed to evaluate regional cerebral perfusion. Given the fact that cerebral blood flow is closely linked to neuronal activity, the radionuclide distribution is presumed to reflect neuronal activity levels in different areas of the brain. A lipophilic, PH-neutral radiopharmaceutical 99m Tchexamethylpropyleneamineoxime (HMPAO) is injected into the patient's blood-flow, crossing the blood-brain barrier while emitting gamma rays. A 3-dimensional representation of cerebral blood flow can be iterated using gamma detectors, allowing for interpretation. Brain SPECT imaging has many different clinical applications ranging from endocrine radionuclide scintigraphy, analysis in major psychiatric disorders or assessment of inflammation in patients with cerebral ischemia (Van Der Naalt 2015).

\section{Design}

We performed a retrospective analysis of $\mathrm{AD}, \mathrm{VaD}$ and MCI patients, examining EEG and SPECT images that were collected as part of clinical diagnostic procedure.

\section{SUBJECTS AND METHODS}

The retrospective analysis was approved by the University Hospital Center Zagreb Medical Research Ethical Committee. The analysis included the group of patients diagnosed with $\mathrm{AD}, \mathrm{VaD}$ and $\mathrm{MCI}$. The study group consisted of 50 patients - 29 females and 21 males. The group of patients diagnosed with $\mathrm{AD}$ was comprised of ten female and nine male patients, with the average age of 77.2 years for women and 77.8 years for men. The group of patients diagnosed with $\mathrm{VaD}$ consisted of eight female and three male patients, with the average age of 75 years for women and 80 years for men. The group of patients diagnosed with MCI consisted of eleven female and nine male patients, the average age of women 67.5 years and men 71.5 years. The age and gender distribution, together with the level of education for each patient id presented in Tables 1 and 2. The diagnosis was made according to the NINCDS-ADRDA and DSM-IV diagnostic criteria by a neurologist (Jack 2006) at the Department of Neurology University Hospital Center Zagreb.

The EEG recording was performed utilizing the Nihon Kohden EEG machine under the standardized conditions. Tin electrodes with electrode caps were positioned in 19 scalp sites (FP1, FP2, F3, F4, Fz, F7, F8, C3, C4, Cz, T3, T4, T5, T6, P3, P4, Pz, O1, and O2) according to the international $10-20$ system and were referenced to electronically linked earlobes (A1 and A2).

Brain perfusion single-photon emission computed tomography (SPECT) imaging was performed in Department of Nuclear Medicine University Hospital Center Zagreb. The diagnostic procedure was performed according to the protocol using neutral radiopharmaceutical 99m Tc-hexamethylpropyleneamineoxime (HMPAO).

\section{RESULTS}

In this cross sectional study retrospective analysis included the group of patients diagnosed with AD, $\mathrm{VaD}$ and MCI. The study group was consisted by 50 patients - 29 females and 21 male. All dementia patients were distinguished from MCI and met the diagnostic criteria according to the Diagnostic and Statistical Manual of Mental Disorders, fourth edition (APA 1994), and the $\mathrm{AD}$ and $\mathrm{VaD}$ criteria of the National Institute of Neurologic and Communicative Disorders and Stroke and the Alzheimer's Disease and Related Disorders Association (NINDS-ADRDA), (McKahn et al. 1984).

Table 1. Female patients (age, level of education, diagnosis)

\begin{tabular}{lccc}
\hline Diagnosis & Alzheimer disease & Vascular dementia & Mild Cognitive Impairment \\
\hline The average age & 77.2 & 75.0 & 67.5 \\
Level of education & & & 1 \\
Primary school & 5 & 4 & 8 \\
Secondary school & 4 & 3 & 2 \\
University & 1 & 1 & \\
\hline
\end{tabular}

Table 2. Male patients (age, level of education, diagnosis)

\begin{tabular}{lccc}
\hline Diagnosis & Alzheimer disease & Vascular dementia & Mild Cognitive Impairment \\
\hline The average age & 77.8 & 80.0 & 71.5 \\
Level of education & & & 1 \\
Primary school & 2 & 3 & 6 \\
Secondary school & 4 & & 2 \\
University & 3 & & \\
\hline
\end{tabular}


Table 3. EEG changes in $\mathrm{AD}, \mathrm{VaD}$ and $\mathrm{MCI}$

\begin{tabular}{lccccc}
\hline EEG changes & Normal & $\begin{array}{c}\text { Alpha rhythm- } \\
\text { low frequency }\end{array}$ & Theta waves & $\begin{array}{c}\text { Focal } \\
\text { abonormalities }\end{array}$ & $\begin{array}{c}\text { Spike-and-waves } \\
\text { complex }\end{array}$ \\
\hline Alzheimer disease & 0 & 10 & 19 & 6 & 6 \\
Vascular dementia & 0 & 1 & 11 & 0 & 1 \\
Mild Cognitive Impairment & 9 & 0 & 11 & 0 & 0 \\
\hline
\end{tabular}

Table 4. CBF abnormalities in $\mathrm{AD}, \mathrm{VaD}$ and $\mathrm{MCI}$

\begin{tabular}{lcccccc}
\hline SPECT abnormalities & Normal & $\begin{array}{c}\text { Unilateral } \\
\text { frontal }\end{array}$ & $\begin{array}{c}\text { Unilateral } \\
\text { temporal }\end{array}$ & $\begin{array}{c}\text { Bilateral } \\
\text { temporal }\end{array}$ & $\begin{array}{c}\text { Bilateral } \\
\text { parietal }\end{array}$ & $\begin{array}{c}\text { "Patchy" } \\
\text { abnormalities }\end{array}$ \\
\hline Alzheimer disease & 2 & 1 & 1 & 5 & 6 & 4 \\
Vascular dementia & 3 & 1 & 0 & 0 & 0 & 7 \\
Mild Cognitive Impairment & 9 & 4 & 2 & 0 & 2 & 3 \\
\hline
\end{tabular}

Out of the 19 AD patients, the EEG analysis revealed increased theta waves in the temporal and parietal areas of nineteen patients $(100 \%)$, decreased alpha coherence in the temporal and parietal areas of ten patients $(55 \%)$, spike-and-wave complexes in six patients $(32 \%)$ and focal abnormalities in frontal and temporal regions of six patients (32\%). SPECT analysis in AD patients showed bilateral parietal cerebral blood flow (CBF) abnormality in six patients (32\%), bilateral temporal CBF abnormality of five patients $(26 \%)$, unilateral frontal abnormality in one patient $(5 \%)$, unilateral temporal abnormality in one patient $(5 \%)$, "patchy" abnormalities in four patients (21\%) and two patients $(10 \%)$ had normal CBF.

In the group of 11 patients diagnosed with $\mathrm{VaD}$ EEG analysis exhibited increased theta waves in the temporal and parietal areas of eleven patients $(100 \%)$, decreased alpha coherence in the temporal and parietal areas of one patient (9\%) and spike-and-wave complex in one patient (9\%). SPECT analysis showed "patchy" abnormalities in seven patients $(63 \%)$, unilateral frontal $\mathrm{CBF}$ abnormalities in one patient $(9 \%)$ and three patients (27\%) had normal CBF.

The EEG analysis in the group of 20 patients diagnosed with MCI showed increased theta waves in the temporal and parietal areas of eleven patients (55\%), while EEG was normal in nine patients (45\%). SPECT analysis exhibited normal CBF in nine patients $(45 \%)$, four patients $(20 \%)$ had unilateral frontal abnormality, two patients $(10 \%)$ had unilateral temporal abnormality, two patients (10\%) had bilateral parietal abnormality and three patients (15\%) had "patchy" abnormalities. The summary of the EEG and SPECT results is presented in Tables 3 and 4.

Correlation of EEG changes and SPECT perfusion patterns showed partial overlap, especially in AD patients, with majority of patient exhibiting decreased alpha coherence and spike-and-wave complexes affecting mostly temporal and parietal regions (53\%), while SPECT analysis revealed bilateral parietal cerebral blood flow (CBF) abnormality in six patients (32\%) and bilateral temporal CBF abnormality of five patients (26\%), indicating affection of the same cerebral regions. The major difference between $\mathrm{MCI}$ and $\mathrm{AD}$ patients were the high proportion of patients with normal EEG (45\%) and SPECT imaging (45\%), while theta waves in parietal and temporal regions observed in $55 \%$ of patients could indicate initial changes correlating to putative cognitive decline. Varying finding in the SPECT analysis of MCI patients may be due to the varying underlying cause, namely all of the MCI patients will not progress to a typical $\mathrm{AD}$ pathology.

\section{DISCUSSION}

Clinical electroencephalography is a relatively simple and inexpensive diagnostic tool with a high sensitivity for diffuse organic encephalopathy of various etiologies and with mounting evidence about the specificity for the type of dementia (Garn et al. 2017). EEG plays an important role in evaluating brain activity. This study is focused on using EEG as a method to detect dementia in the early stages. There is an urgent need for an accurate, specific, and costeffective method for early dementia diagnosis. This makes EEG an attractive tool to detect and differentiate $\mathrm{AD}$ and $\mathrm{VaD}$ in the early stages due to its affordability and noninvasiveness. EEG evaluation through visual inspection is prone to mistakes due to the subjective experience of neurologists (Al-Oazzaz et al. 2014). EEG is the only diagnostic instrument directly reflecting cortical neuronal functioning and it remains an important clinical tool in the diagnosis and differential diagnosis of AD. Although a normal EEG is found in many patients with mild $\mathrm{AD}$, a pathological EEG is an important finding, because such a result is not expected in differential diagnoses such as depression or pseudodementia within a dissociative disorder. Epileptic activity associated with $\mathrm{AD}$ is a well-known fact which deserves increased attention because it can have an adverse impact on the patients, is often overlooked and untreated, and may be linked to pathological occurrences leading to worsening of the illness. The fact that the vast majority of patients with moderate to severe AD exhibit a pathological EEG may in part be due to the epileptogenic changes occurring in 
those patient, but cannot fully explain the specific changes in the EEG. A normal EEG in this group of patients is more common in subcortical dementia or frontal lobe degeneration than AD. Compared to SPECT or routine structural brain imaging (CT, MRI), EEG has a comparable diagnosis sensitivity and a higher specificity. For monitoring changes of brain function by serial recordings (e.g., during therapy with anti-dementia drugs), EEG is the best available method (Al-Qazzaz et al. 2014). Using brain SPECT imaging, a nuclear medicine study that measures blood flow and activity, researchers found that people with cognitive disorders had specific patterns of reduced CBF in multiple brain areas compared to those with depression, particularly in the hippocampus, temporal, and parietal lobes. They also found that SPECT could distinguish depression from dementia with $86 \%$ accuracy. In addition, brain SPECT imaging showed the ability to distinguish depression or dementia in people with both with $83 \%$ accuracy (Amen et al. 2017).

The results of our study indicate that in the group of patients with AD EEG changes are present in all patients. These changes are mostly in the form of theta waves, focal abnormalities and spike-and- wave complexes in frontotemporal regions with the reduction amplitude of alpha waves. SPECT showed hypoperfusion in temporoparietal regions with occasional unilateral abnormalities in frontotemporal region. EEG changes in patients with $\mathrm{VaD}$ were predominantly in the form of theta waves while SPECT finds mostly "patchy" abnormalities. EEG readings were normal or exhibit minimal changes in the group of patients with MCI, while SPECT imaging exhibits mostly normal CBF. These results confirm previous studies, which have shown that patients at high risk for developing AD have reduced SPECT perfusion in the temporoparietal regions and inferior parietal lobule (DeCarli et al. 2005, Pennanen et al. 2011).

The common underpinning for the electrophysiological and perfusion changes could be found in the previous studies aimed at discovering specific patterns in patients with AD. Previous studies have shown an increase in delta and theta activity as well as reduction of alpha and beta activity, especially in posterior regions, which correlates with our findings (Tsolaki et al. 2014). Several studies have tried to correlate the imaging findings with EEG readings. Babiloni et al. exhibited that resting state EEG abnormalities in AD and MCI occur because of the specific patterns of brain atrophy (Babiloni et al. 2013). In an effort to develop a novel tool for EEG analysis, DIMENSION method was developed which also showed that SPECT readings in $\mathrm{AD}$ patients with parietal hypoperfusion had decreasing mean value and increasing standard deviation of EEG alpha dipolarity (Kouzuki et al. 2013). The global decrease in cerebral blood flow $(\mathrm{CBF})$ is associated with a shift on the topographical alpha-centroids in the posterior direction. The correlation between $\mathrm{CBF}$ and clinical severity is weak, indicating a high interindividual variance or interactions with concomitant vascular lesions (Müller et al. 1997).

More recent studies have also tried to correlate patterns of hypometabolism as measured by PET with specific EEG findings. One such study has utilized the so-called Alzheimer's discrimination analysis tool (PALZ) and showed that patients with AD exhibited higher activity of delta sources and lower activity of low-frequency alpha sources in specific cortical regions, pointing to a relationship between cortical hypometabolism and delta rhythm neuronal synchronization in dementia patients (Babiloni et al. 2016). Collective data point to the fact that changes in EEG readings most probably stem from the specific patterns of brain atrophy in patients with $\mathrm{AD}$, which is also reflected in patterns of hypoperfusion as depicted by SPECT. On a molecular level, specific EEG alterations also stem from the changes in the metabolic status of the cortical neurons, thus reflecting the overall functioning of the brain parenchyma.

Based on the presented results, we propose that EEG can be used to support the diagnosis, monitor the therapeutic effect and possibly prognosticate the course of the disease, especially in patients with $\mathrm{AD}$. In the patients with the MCI EEG may be used to monitor the conversion to the early stage AD.

The limitations of the present study are the small number of patients with $\mathrm{AD}, \mathrm{VaD}$ and $\mathrm{MCI}$, and lack of additional correlation studies with more advanced neuroimaging analyses, such as FDG-PET.

\section{CONCLUSION}

Various diagnostic procedures are used in the diagnosis of dementia. EEG and SPECT are diagnostic methods that show specific changes, especially in AD. EEG can be used to monitor the therapeutic effect and progression of $\mathrm{AD}$ as well as the possible transition from MCI to early stage AD. SPECT on the other hand, being a more expensive and less available method, can be utilized as an add-on method to increase the specificity and sensitivity of the diagnostic algorithm.

Acknowledgements: None.

Conflict of interest: None to declare.

\section{Contribution of individual authors:}

Borislav Radić \& Fran Borovečki designed and wrote work.

Ratimir Petrović \& Anja Golubić processed the data. Ervina Bilić wrote the final opinion. 


\section{References}

1. Al-Qazzaz NK, Bin SH, Ahmad SA, Chellappan K, Islam $S H$ and Escudero J: Role of EEG as Biomarker in the Early Detection and Classification of Dementia. The Scientific World Journal Volume 2014, Article ID 906038, 16 pages

2. Amen DG, Krishnamani P, Meysami S, Newberg A, Raji CA: Classification of Depression, Cognitive Disorders, and Co-Morbid Depression and Cognitive Disorders with Perfusion SPECT Neuroimaging. J Alzheimers Dis 2017. doi: 10.3233/JAD-161232

3. APA: Diagnostic and Statistical Manual of Mental Disorders. IV ed. Washington, DC: American Psychiatric Press, 1994

4. Babiloni C, Carducci F, Lizio R, Vecchio F, Baglieri A, Bernardini S, Cavedo E, Bozzao A, Buttinelli C, Esposito $F$, Giubilei $F$, Guizzaro A, Marino $S$, Montella $P$, Quattrocchi CC, Redolfi A, Soricelli A, Tedeschi G, Ferri $R$, Rossi-Fedele G, Ursini $F$, Scrascia F, Vernieri $F$, Pedersen TJ, Hardemark HG, Rossini PM, Frisoni GB. Resting state cortical electroencephalographic rhythms are related to gray matter volume in subjects with mild cognitive impairment and Alzheimer's disease. Hum Brain Mapp 2013; 34:1427-46

5. Babiloni C, Del Percio C, Caroli A, Salvatore E, Nicolai E, Marzano N, Lizio R, Cavedo E, Landau S, Chen K, Jagust W, Reiman E, Tedeschi G, Montella P, De Stefano M, Gesualdo L, Frisoni GB, Soricelli A: Cortical sources of resting state EEG rhythms are related to brain hypometabolism in subjects with Alzheimer's disease: an EEGPET study. Neurobiol Aging 2016; 48:122-134

6. Blenow $K$, de Leon MJ, Zetterberg H: Alzheimer's disease. Lancet 2006; 368:387-403

7. Burns A, Iliffe S: Alzheimer's disease. The BMJ 2009; 338:b158

8. Cichocki A, Shishkin SL, Musha T, Leonowicz Z, Asada T, Kurachi T: EEG filtering based on blind source separation (BSS) for early detection of Alzheimer's disease. Clin Neurophysiol 2005; 116:729-37

9. DeCarli C, Fletcher E, Ramey V, Harvey D, Jagust WJ: Anatomical mapping of white matter hyperintensities $(W M H)$ : exploring the relationships between periventricular WMH, deep WMH, and total WMH burden. Stroke 2005; 36:50-55

10. DeKosky ST, Marek K: Looking backward to move forward: early detection of neurodegenerative disorders. Science 2003; 302:830-4

11. Dierks T, Frölich L, Ihl R, Maurer K: Correlation between cognitive brain function and electrical brain activity in dementia of Alzheimer type. J Neural Transm Gen Sect 1995; 99:55-62
12. Garn H, Coronel C, Waser M, Caravias G, Ransmayr G: Differential diagnosis between patients with probable Alzheimer's disease, Parkinson's disease dementia, or dementia with Lewy bodies and frontotemporal dementia, behavioral variant, using quantitative electroencephalographic features. J Neural Transm (Vienna) 2017; 124:569-581

13. Kouzuki M, Asaina F, Taniguchi M, Musha T, Urakami K: The relationship between the diagnosis method of neuronal dysfunction (DIMENSION) and brain pathology in the early stages of Alzheimer's disease. Psychogeriatrics 2013; 13:63-70

14. Kwak YT: Quantitative EEG findings in different stages of Alzheimer's disease. J Clin Neurophysiol 2006; 23:456-61

15. Jack CR, Albert MS, Knopman DS, McKhann GM, Sperling RA, Carrillo MC, Thies B, Phelps CH: Introduction to the recommendations from the National Institute on Aging-Alzheimer's Association workgroups on diagnostic guidelines for Alzheimer's disease. Alzheimers Dement 2011; 7:257-262

16. McKhann G, Drachman D, Folstein M, Katzman R, Price D, Stadlan EM: Clinical diagnosis of Alzheimer's disease: report of the NINCDS-ADRDA Work Group under the auspices of Department of Health and Human Services Task Force on Alzheimer's Disease. Neurology 1984; 34:939-44

17. Müller TJ, Thome J, Chiaramonti R, Dierks T, Maurer K, Fallgatter AJ, Frölich L, Scheubeck M, Strik WK: A comparison of qEEG and HMPAO-SPECT in relation to the clinical severity of Alzheimer's disease. Eur Arch Psychiatry Clin Neurosci 1997; 247:259-63

18. Pennanen C, Testa C, Laasko MP, Hallikainen M, Helkala EL, Hanninen T, et al: A voxel based morphometry study on mild cognitive impairment. J Neurol Neurosurg Psychiatry 2005; 76:11-14

19. Psota G: Social psychiatric aspect of dementia. Psychiatr Danub 2015; 27:432-438

20. Roman GC: Vascular dementia revisited: diagnosis, pathogenesis, treatment, and prevention. Med Clin North Am 2002; 86:477-99

21. Tsolaki A, Kazis D, Kompatsiaris I, Kosmidou V, Tsolaki M: Electroencephalogram and Alzheimer's Disease: Clinical and Research Approaches. Int J Alzheimers Dis 2014; 2014:349249

22. Van Der Naalt J: Resting functional imaging tools (MRS,SPECT, PET and PCT). Handbook of Clinical Neurology 2015; 127:295-308

23. Wancota J, Freidl M, Unger A, Jahn R, Soulier N, Fellinger $M \&$ Daniel $R$ : Projections about the future number of dementia suffers: increasing life expectancy not sufficiently considered? Psychiatr Danub 2015; 27:452-457

24. Weichbold B: Careways of dementia care. Psychiatr Danub 2015; 27:439-445

Correspondence:

Borislav Radić, $M D$

Department of Neurology, University Hospital Center Zagreb

Kišpatićeva 12, 10000 Zagreb, Croatia

E-mail:boris.radic105@gmail.com 